\title{
Tuning Spontaneous Emission through Waveguide Cavity Effects in Semiconductor Nanowires
}

Florian Dirnberger, ${ }^{\dagger} \odot$ Diego Abujetas, ${ }^{\ddagger}$ Jan König, ${ }^{\dagger}$ Moritz Forsch, ${ }^{\dagger}$ Thomas Koller, $^{\dagger}$ Imke Gronwald, $^{\dagger}$ Christoph Lange, ${ }^{\dagger}$ Rupert Huber, ${ }^{\dagger}$ Christian Schüller, ${ }^{\dagger}$ Tobias Korn, ${ }^{\S}$ José Sánchez-Gil, ${ }^{\ddagger}{ }^{\ddagger}$ and Dominique Bougeard ${ }^{*} \dagger$ (0)

${ }^{\dagger}$ Institut für Experimentelle und Angewandte Physik, Universität Regensburg, D-93040 Regensburg, Germany

${ }^{\ddagger}$ Instituto de Estructura de la Materia (IEM-CSIC), Consejo Superior de Investigaciones Científicas, Serrano 121, 28006 Madrid, Spain

${ }^{\S}$ Institut für Physik, Universität Rostock, D-18051 Rostock, Germany

Supporting Information

ABSTRACT: The ability to tailor waveguide cavities and couple them with quantum emitters has developed a realm of nanophotonics encompassing, for example, highly efficient single photon generation or the control of giant photon nonlinearities. Opening new grounds by pushing the interaction of the waveguide cavity and integrated emitters further into the deep subwavelength regime, however, has been complicated by nonradiative losses due to the increasing importance of surface defects when decreasing cavity dimensions. Here, we show efficient suppression of nonradiative recombination for thin waveguide cavities using core-shell semiconductor nanowires. We experimentally reveal the advantages of such nanowires, which host mobile emitters, that is, free excitons, in a one-dimensional (1D) waveguide, highlighting the resulting potential for tunable, active, nanophotonic devices. In our

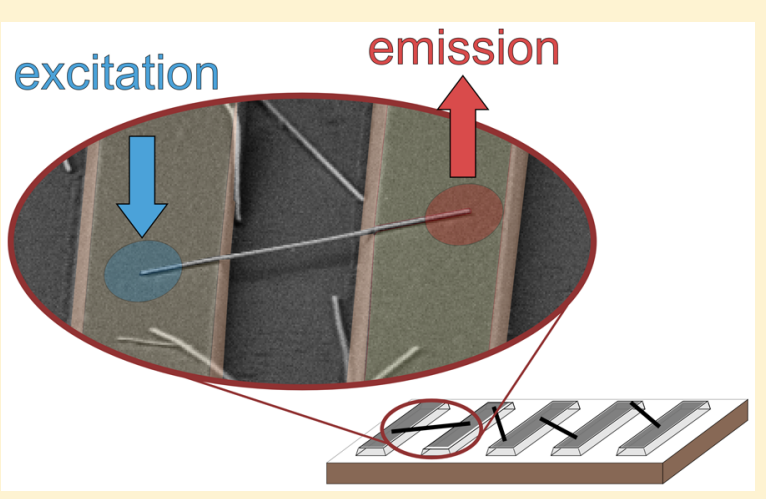
experiment, controlling the nanowire waveguide diameter tunes the luminescence lifetime of excitons in the nanowires across 2 orders of magnitude up to $80 \mathrm{~ns}$. At the smallest wire diameters, we show that this luminescence lifetime can be manipulated by engineering the dielectric environment of the nanowires. Exploiting this unique handle on the spontaneous emission of mobile emitters, we demonstrate an all-dielectric spatial control of the mobile emitters along the axis of the $1 \mathrm{D}$ nanowire waveguide.

KEYWORDS: Semiconductor nanowire, luminescence lifetime, tunable spontaneous emission, photonic waveguide cavity, free excitons, subwavelength nanophotonics

$\mathrm{T}$ the past decade, semiconductor nanowires (NWs) have been established as a fundamental building block of nanophotonics in highly efficient light-emitting diodes or solar cells, ${ }^{1,2}$ as the active component of nanoscale lasers and terahertz detectors, ${ }^{3,4}$ or as a passive element in novel photochemical sensors. ${ }^{5}$ The photocarrier recombination dynamics of such NWs has been broadly investigated, finding that due to the large surface-to-volume ratio these dynamics are mostly dominated by nonradiative recombination at defects $^{6,7}$ or surfaces ${ }^{8-10}$ and by electric field-induced band bending effects. ${ }^{11,12}$ Although a significant impact of waveguide cavity effects on the interaction of light with semiconductor NWs would be expected for the diameter range of typical vapor-liquid-solid-grown $\mathrm{NWs}^{13}{ }^{13}$ a few tens to hundreds of nanometers, their manifestation in the photocarrier recombination dynamics has not been considered, besides in NW lasing studies, ${ }^{3,14,15}$ mostly as a consequence of the significant nonradiative recombination.
In this Letter, we present defect-free core/shell NWs for which we experimentally show the nonradiative effects to be negligible. For these wires, we then demonstrate that the waveguide cavity effects completely dominate the recombination process, resulting in a strong dependence of the photocarrier dynamics on the NW diameter: The luminescence lifetime of the observed free exciton recombination varies over 2 orders of magnitude, in good agreement with our simulations. As a second important manifestation of these cavity effects, we evidence a pronounced impact of the dielectric environment which surrounds a single NW (vacuum or dielectric substrate) on the free exciton recombination in the thinnest wires. Taking advantage of this effect, we locally engineer the dielectric environment of individual NWs,

Received: July 15, 2019

Revised: September 9, 2019

Published: September 16, 2019 
allowing us to demonstrate all-dielectric spatial control over the free exciton emission along the axis of a single wire.

The GaAs $/ \mathrm{Al}_{0.36} \mathrm{Ga}_{0.64}$ As core/shell NWs are synthesized by molecular beam epitaxy from a Au-seeded growth on GaAs(111)B substrates. In a series of wafers, we tune the core diameter of a NW ensemble before capping the NWs with a $10 \mathrm{~nm}$ thin $\mathrm{Al}_{0.36} \mathrm{Ga}_{0.64}$ As shell. Because of the larger band gap, the shell confines all photocarriers to the NW core. Finally, a $5 \mathrm{~nm}$ thin GaAs layer is grown to avoid oxidation of the shell. The nominally undoped NWs grow vertically on the growth substrate and adopt the wurtzite crystal phase of GaAs with very high phase purity. ${ }^{16}$ In the following, the diameter $d$ refers to the total diameter of the NW as measured between two opposite corners of the hexagonal cross-section. Our series covers NW diameters in the range of $d=530$ to $60 \mathrm{~nm}$ whereas their length decreases from $\sim 6$ to $1 \mu \mathrm{m}$ as the diameter decreases. ${ }^{17}$

As sketched in the central part of Figure 1, we excite single wires in a confocal microphotoluminescence $(\mu$-PL) setup at

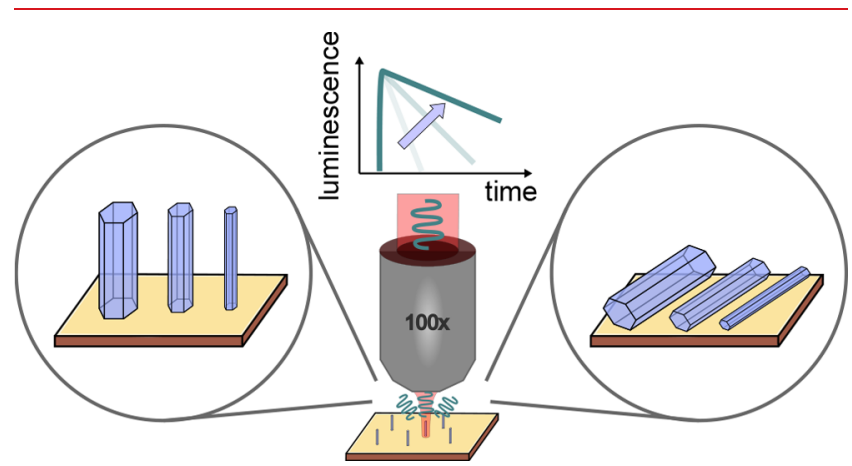

Figure 1. Simplified schematic of the exciton dynamics study in our NWs. A pulsed laser is focused by a $100 \times$ microscope objective to excite single wires and record their luminescence dynamics (top). NWs in a large range of diameters are investigated either free-standing (left) or lying on a $\mathrm{Si} / \mathrm{SiO}_{2}$ substrate (right).

nominally $4.2 \mathrm{~K}$ using a near-resonant $(1.58 \mathrm{eV})$ laser diode with 70-500 ps pulses at a repetition frequency of $1 \mathrm{MHz}$ and detect their luminescence dynamics in a streak camera system. A $100 \times$ microscope objective focuses the laser beam to a spot size of $\sim 1 \mu \mathrm{m}^{2}$, allowing us to investigate single NWs either directly as-grown when they are free-standing on the growth substrate $^{18}$ or after they were dispersed onto a substrate composed of $300 \mathrm{~nm} \mathrm{SiO}$ on $\mathrm{Si}$ (see left and right panels in Figure 1). The diameter-dependent absorption of light in our wires $^{19,20}$ requires an adjustment of the laser power with decreasing diameter. For free-standing NWs with $d=530$ to $150 \mathrm{~nm}$, we find a negligible variation of the decay time of photoexcited carriers when varying the excitation power density by a factor of 25 . For $d=530-150 \mathrm{~nm}$, we thus use a time-averaged pulsed excitation power density of $1.4 \mathrm{~W} / \mathrm{cm}^{2}$, which we gradually increase up to $267 \mathrm{~W} / \mathrm{cm}^{2}$ for wires with $d$ $<150 \mathrm{~nm}$ to compensate for their reduced absorption. In Supporting Information SI1, we estimate a photoexcited carrier density $n \approx 10^{17} \mathrm{~cm}^{-3}$ right after the pulsed excitation, which is only slightly above the metal-insulator (or Mott-) transition; ${ }^{21,22}$ the dynamics at later times is then governed by excitonic properties. We thus regard the photoexcited carriers in our NWs as a gas of free and mobile excitons. Their luminescence decay is obtained directly from the time-resolved streak camera images by spectrally integrating over a $5 \mathrm{meV}$ wide window centered at the peak of the PL emission.

Prior to the time-resolved experiments, we preselect NWs showing a defect-free optical emission associated with high phase purity. ${ }^{16,18,23}$ As demonstrated in Supporting Information SI2, our selection process sorts out defect-afflicted NWs and ensures that we observe the dynamics of free and delocalized excitons in our NWs.

First, we focus on the free exciton dynamics in the freestanding NWs. We have divided their diameter-dependence into two plots, Figure 2a,b, with different scalings of the time

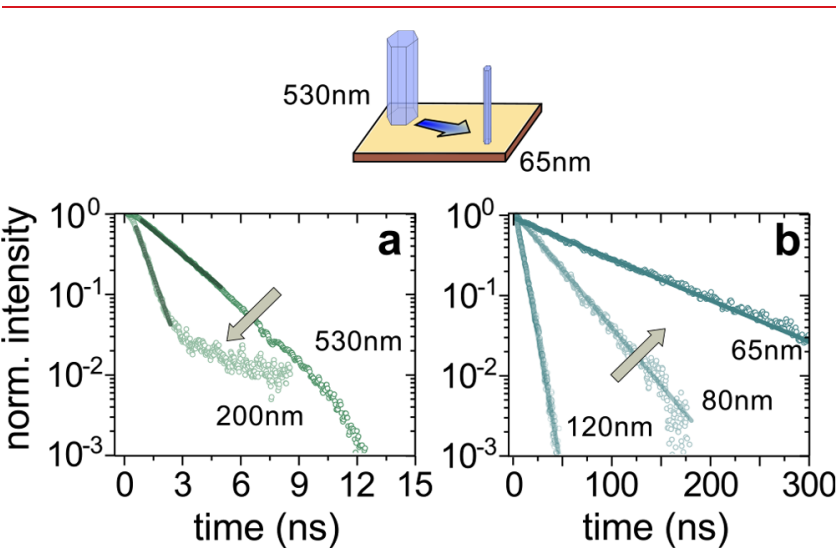

Figure 2. Normalized low-temperature luminescence decay curves of single free-standing NWs. (a,b) The remarkable differences of the exciton recombination dynamics in free-standing NWs with diameters ranging from $d=530$ to $65 \mathrm{~nm}$. The fits are plotted as solid lines. Note the different time scales in $(a, b)$.

axis. Figure 2a compares the normalized decay curves of two of the larger diameters, $d=530$ and $200 \mathrm{~nm}$. The major part of the total luminescence intensity $(>90 \%)$ can be well characterized by a single exponential decay lifetime for both diameters. From the fits plotted as solid lines, we find $\tau=2.1$ $\mathrm{ns}$ for $d=530 \mathrm{~nm}$ and $\tau=0.6 \mathrm{~ns}$ for $d=200 \mathrm{~nm}$. Hence, the luminescence decays by up to a factor of 3.5 faster in the NW with $d=200 \mathrm{~nm}$ as compared to the thicker NW.

Strikingly, this trend reverses strongly toward smaller diameters. Decreasing the diameter from $d=200$ to $120 \mathrm{~nm}$ between Figure 2a,b, we already observe a 10 -fold increase in the luminescence lifetime to $\tau=6 \mathrm{~ns}$. Figure $2 \mathrm{~b}$ then shows three NWs with the diameters $d=120,80$, and $65 \mathrm{~nm}$ for which the traces are now fully described by a single exponential decay. The luminescence lifetimes keep increasing for the two smaller diameters, yielding the surprisingly long lifetime $\tau=86$ $\mathrm{ns}$ for $d=65 \mathrm{~nm}$. Because most reports on the luminescence dynamics of semiconductor NWs demonstrate lifetimes in the picosecond to nanosecond range, ${ }^{6-9,12,23,24}$ observing such markedly long lifetimes in our NWs is intriguing.

To provide an overview of the diameter dependence, we averaged the lifetimes $\bar{\tau}$ from several single wires with nominally identical diameters and summarized the results for a range of NW diameters from $d=530$ to $60 \mathrm{~nm}$ in Figure 3a. The graph confirms the trends observed in Figure $2 \mathrm{a}, \mathrm{b}$ and shows two main features:

(i) For $d \geq 150 \mathrm{~nm}$, the average lifetimes slightly decrease from $\bar{\tau}=2.1 \mathrm{~ns}$ toward a minimum around $d=200 \mathrm{~nm}$. Comparatively, these lifetimes depend only weakly on the NW diameter. 


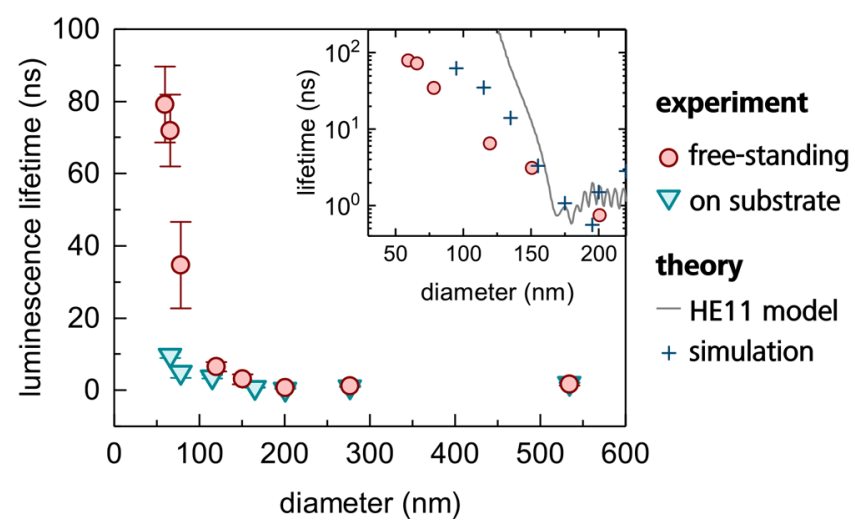

b
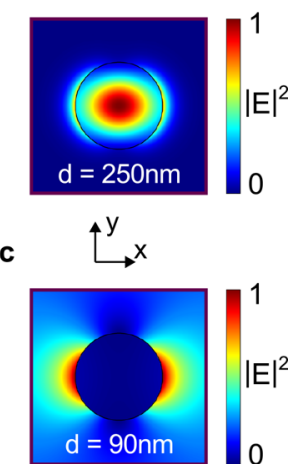

Figure 3. (a) Diameter-dependence of the averaged luminescence lifetime $\bar{\tau}$ in our NWs. Upon decreasing the NW diameter, the measurements of free-standing wires (red circles) reveal a tremendous increase in the luminescence lifetime for $d \leq 150$ nm. For these diameters, the NWs lying on the $\mathrm{Si} / \mathrm{SiO}_{2}$ substrate (blue triangles) show significantly shorter lifetimes. The error bars denote the statistical standard deviation. Solid gray line and blue crosses in the inset represent the lifetime results of our 1D current model and the COMSOL simulations. (b,c) Real space distribution of the $\mathrm{HE}_{11}$ mode in a wire with $d=250 \mathrm{~nm}$ and $d=90 \mathrm{~nm}$.

(ii) For $d<150 \mathrm{~nm}$, however, a dramatic increase in the lifetimes occurs, culminating at an average of $\bar{\tau}=79 \mathrm{~ns}$ for the thinnest NWs $(d=60 \mathrm{~nm})$.

Remarkably, the time over which the absorbed photon energy is stored in the excitons in a NW before being reemitted is thus tuned over 2 orders of magnitude in our NWs through the choice of the diameter, posing the question which microscopic process determines the decay? Because free excitons occupy the whole NW volume in less than 200 $\mathrm{ps}^{25}$ exciton diffusion does not contribute to the luminescence decay at later times. Therefore, only radiative and nonradiative processes need to be considered here. We discuss recombination rates induced by nonradiative sources in detail in Supporting Information SI3. From our experimental observations, we deduce that we can exclude nonradiative recombination by Auger processes, as well as phonon-induced recombination at crystal point defects in the GaAs core. In contrast to wires without a shell, ${ }^{8}$ surface recombination is also not relevant for our $\mathrm{GaAs} / \mathrm{Al}_{0.36} \mathrm{Ga}_{0.64} \mathrm{As}$ core/shell NWs. We note that any nonradiative process related to surface recombination would yield a significant decrease of the observed luminescence lifetime, contrary to our experimental observations, as the surface-to-volume ratio increases with decreasing NW diameter. Because all relevant sources of nonradiative recombination as well as diffusion effects do not dominantly contribute to the observed dynamics, we conclude that we probe the radiative decay of free excitons as the main recombination channel. Hence resulting from the spontaneous emission process of free excitons, the NW luminescence now provides an opportunity to experimentally study the intrinsic exciton emission dynamics in a direct band gap semiconductor NW.

Note that compared to the moderate width-dependence of the spontaneous emission of free excitons in two-dimensional $\mathrm{GaAs} / \mathrm{AlGaAs}$ quantum wells, ${ }^{26}$ the diameter-dependence of the lifetime observed in Figure 3 is dramatic. Only specifically designed microcavities, such as photonic crystals or plasmonic structures, have been shown to allow such drastic variations in the radiative lifetimes of single emitters. ${ }^{2728-30}$ In the following, we will show that our results are very well described by considering the impact of the NW waveguide cavity on the spontaneous emission of free excitons.
Starting by modeling a dipole emitter located in a dielectric waveguide, it is found to be forced to radiate its energy into the optical modes of the waveguide. ${ }^{13,31,32}$ Particularly, for cylindrical dielectric waveguides with a refractive index $n=$ 3.45 , corresponding to GaAs, the fundamental $\mathrm{HE}_{11}$ mode of the $\mathrm{NW}$ waveguide dominates the mode spectrum in the diameter-regime around $d=150 \mathrm{~nm} \cdot{ }^{31-33}$ Here, we model the role of the $\mathrm{HE}_{11}$ mode in the spontaneous emission process of excitons in our NWs by using a 1D current model, ${ }^{34}$ which describes the NW as a one-dimensional cavity in vacuum with length $L$. In this parameter-free model, the emission is produced by a line current which is excited by a dipole at a given position along the wire (see Supporting Information SI4). The model allows us to calculate the power radiated to the far-field from the $\mathrm{HE}_{11}$ mode, this power being directly proportional to the spontaneous emission. We have plotted the lifetime deduced from this modeled fundamental $\mathrm{HE}_{11}$ mode together with the experimentally obtained lifetimes in the inset of Figure 3a on a semilogarithmic scale. Qualitatively, just like in the experiment, the lifetimes derived from our 1D current model also show a weak diameter-dependence for larger diameters, contrasting with a very steep increase setting on at $d$ $\approx 150 \mathrm{~nm}$. As can be noted in the inset of Figure $3 \mathrm{a}$, the slope of the increase is steeper for the model than for the experimental data. We attribute this difference to the fact that although the $\mathrm{HE}_{11}$ mode dominates the mode spectrum, the free excitons in our NWs still also weakly couple their emission into the residue of a continuum of free space modes, which is not accounted for by the $1 \mathrm{D}$ current model. This effect becomes more pronounced precisely as the NW diameter is decreased. To take this effect into account, we have additionally numerically simulated the lifetimes of on-axis emitters in COMSOL (see Supporting Information SI4), which considers the emission into all available modes. The result of this parameter-free simulation is plotted as blue crosses in the inset of Figure $3 a$ and leads to a very good agreement with the experimental data. We thus conclude that the spontaneous emission in our NWs is highly sensitive to waveguide cavity effects which are governed by the NW diameter. Note that this effect is most pronounced in a diameter-range ( $d=60$ to $150 \mathrm{~nm}$ ) that is typical for vaporliquid-solid grown semiconductor NWs. In particular, the 
spontaneous emission is inhibited by up to 2 orders of magnitude for small diameters, more strongly than previously reported for single quantum dot emitters studied in photonic wires. $^{31,35}$

Plotting the real-space distribution of the $\mathrm{HE}_{11}$ mode intensity, in Figure 3a,b, provides an illustrative account of the diameter-dependent lifetime effects in our experiment. For a thicker wire with $d=250 \mathrm{~nm}$, the major part of the $\mathrm{HE}_{11}$ mode intensity is confined within the NW (see Figure $3 \mathrm{~b}$ ). For smaller wire diameters, however, it can clearly be seen in Figure $3 \mathrm{c}$ that the $\mathrm{HE}_{11}$ mode gets more and more expelled out of the NW, so that the largest fraction of the mode intensity is actually redistributed to the direct environment (here vacuum) of the NW, thus strongly reducing the photonic local density of states inside. It is then clear that the small mode intensity within the GaAs/AlGaAs NW material strongly reduces the spontaneous emission probability of excitons and is eventually the reason for the long luminescence lifetimes in the thin NWs.

Transposing the idea of an expelled optical mode to our experiment, we anticipate a considerable impact on the spontaneous emission of excitons when we modify the direct environment of our small-diameter NWs, for example, by depositing them onto a dielectric substrate instead of probing free-standing NWs in vacuum. We indeed clearly observe this impact in Figure $3 \mathrm{a}$ where we compare the diameterdependent luminescence lifetimes of NWs lying on $\mathrm{Si} / \mathrm{SiO}_{2}$ substrates with the free-standing NWs. In agreement with the mode distribution illustrated in Figure $3 \mathrm{~b}$, the lifetimes of the largest NWs, where the optical modes are confined within the NW, are identical in both configurations of the NW environment. In contrast, approaching the small-diameter regime $(d \leq 150 \mathrm{~nm})$ of our NWs introduces a quantitative difference: although the lifetimes of the NWs lying on substrates also increase for decreasing diameters, they are clearly shorter than those of the free-standing NWs. For the thinnest wires of our series, the difference between both configurations reaches up to 1 order of magnitude. We relate this effect to the difference in the refractive index surrounding the NWs, that is, vacuum for the free-standing wires and an increased effective index for $\mathrm{NWs}$ lying on the $\mathrm{Si} / \mathrm{SiO}_{2}$ substrate. $^{36}$

This strong dependence of the spontaneous emission of free excitons on the direct environment of a small-diameter NW offers an interesting tuning knob on the spontaneous emission: A local manipulation of the dielectric environment should allow us to tune the luminescence lifetime along the axis of a single wire. As a proof of principle, we have fabricated a substrate hosting an array of $\mathrm{SiO}_{2}$ stripes by electron beam lithography, as sketched in Figure 4a. The gap between two neighboring stripes is $\sim 3 \mu \mathrm{m}$. The individual stripes have a height of $450 \mathrm{~nm}$ and a width of $3.3 \mu \mathrm{m}$. The colored scanning electron micrograph in Figure 4a shows a NW suspended between two neighboring stripes (position (1) and (3) in Figure $4 a$ ). In between the stripes, the NW is surrounded by vacuum (position (2). This spatial modulation of the refractive index of the NW environment now enables us to study the effects on the spontaneous emission locally along the axis of a single wire. The corresponding luminescence decay curves plotted in Figure $4 \mathrm{~b}$ unambiguously demonstrate that excitons excited and detected in the vicinity of the $\mathrm{SiO}_{2}$ stripe (1) show a shorter lifetime of $\tau=2.6 \mathrm{~ns}$, whereas in the part of the NW which is surrounded by vacuum (2) the exciton recombination is inhibited, decaying with a longer lifetime of $\tau=4.8 \mathrm{~ns}$.

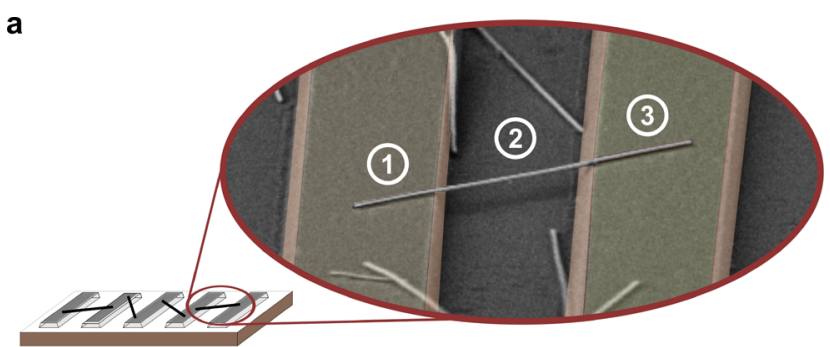

b
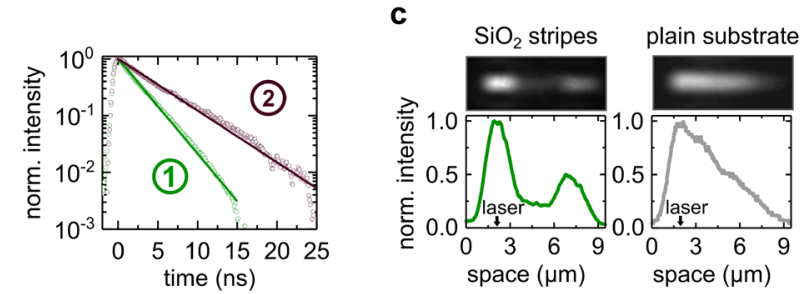

Figure 4. (a) Scanning electron micrograph of a NW supported on both ends by a $\mathrm{SiO}_{2}$ stripe (highlighted by transparent yellow rectangles). In between, a $3 \mu \mathrm{m}$ long section of the wire is surrounded by vacuum. For this experiment, we applied the Ga-assisted growth on $\mathrm{Si}(111)$ substrates to synthesize a new batch of $\mathrm{GaAs} / \mathrm{Al}_{0.36} \mathrm{Ga}_{0.64} \mathrm{As}$ core/shell NWs with predominantly zincblende crystal phase. While maintaining a small diameter of $d=110 \mathrm{~nm}$, the higher growth temperatures allow us to grow $\sim 8 \mu \mathrm{m}$ long NWs, well suited for the micrometer-distances between the stripes. (b) The two luminescence decay curves, measured at the positions marked in (a), directly demonstrate the spatially modulated lifetimes. (c) Microscope images of the PL intensity and the corresponding intensity profiles extracted along the axis of the NW upon local laser excitation at position (1). For reference, the panel on the right shows the spatial PL intensity of a wire from the same wafer, which was placed on a plain $\mathrm{Si} / \mathrm{SiO}_{2}$ substrate. Each profile was normalized to the maximum intensity and the arrows mark the position of the laser excitation spot. For this experiment, the laser energy was $3.1 \mathrm{eV}$.

Probing the luminescence decay therefore confirms our concept of a spatially tunable lifetime.

Our tuning of the luminescence lifetimes along a single NW is then fully consistent with the local luminescence intensity recorded along the NW axis. This is demonstrated in the microscope image in the left panel of Figure 4c. It displays the spatial distribution of the luminescence upon continuously exciting excitons near the left end of the NW (1), which is located on top of the first $\mathrm{SiO}_{2}$ stripe. Although the maximum of the emission intensity occurs directly at the excitation spot, the luminescence signal drops significantly along the NW, where it is surrounded by vacuum. Then, at the opposite end of the NW where the NW lies on top of $\mathrm{a} \mathrm{SiO}_{2}$ stripe (3), a second maximum occurs. This observation cannot be attributed to enhanced scattering of light at the ends of the waveguide, as demonstrated by a control experiment: The right panel of Figure $4 c$ shows the experimental emission profile of a NW similar to the one shown in the left panel, taken from the same growth batch. In contrast to the right panel, this $\mathrm{NW}$ is now lying on an unprocessed $\mathrm{Si} / \mathrm{SiO}_{2}$ substrate in its entire length. Its spatially resolved intensity profile differs from the left panel. It is characterized by a continuously decreasing luminescence intensity starting from the excitation spot, illustrating the diffusion of the photoexcited free excitons in a NW with a constant luminescence lifetime along the whole wire axis. No characteristic influence of light scattering at the ends of the NW on the plain substrate is observed here as a 
result of the small diameters of the NWs for $d \leq 150 \mathrm{~nm} \cdot{ }^{37}$ We also exclude the influence of any structural anisotropies as exciting the NW at the right end (3) spatially inverts the luminescence profile (see Supporting Information SI5). As a consequence, our experiments demonstrate that engineering the dielectric environment of GaAs/AlGaAs core/shell NWs along the wire axis permits a significant spatial tuning of the spontaneous emission at the nanoscale.

In conclusion, our study of the spontaneous emission of free excitons in GaAs NWs unveils a dominant role of the waveguide cavity effects that are inherent to semiconductor NWs. This finding is particularly relevant for core/shell vaporliquid-solid grown NWs because the drastic increase of the luminescence lifetime by 2 orders of magnitude in vacuum falls into the typical wire diameter range of a few tens to a few hundreds of nanometers. Furthermore, entering the smalldiameter regime, the dominating contribution of the fundamental waveguide mode and its pronounced sensitivity on the dielectric surrounding of a NW give access to an interesting tuning knob. We show that bringing a $\mathrm{NW}$ in contact with a $\mathrm{Si} / \mathrm{SiO}_{2}$ substrate reduces the lifetimes up to a factor of 10 compared to a NW surrounded by vacuum. This observation opens perspectives for realizing concepts related to subwavelength and Mie-resonant effects which are broadly studied with dielectric nanoparticles to engineer light-matter interaction at the nanoscale. ${ }^{38}$ As an illustration of these perspectives, we demonstrate that the peculiar interaction of NWs with light enables us to locally control and thus engineer the luminescence lifetime of excitons along the axis of a single wire by patterning the substrate on the nanoscale.

\section{ASSOCIATED CONTENT}

\section{S Supporting Information}

The Supporting Information is available free of charge on the ACS Publications website at DOI: 10.1021/acs.nanolett.9b02883.

Estimation of optically excited carrier density, identification of crystal phase pure NWs in $\mu \mathrm{PL}$ spectroscopy, discussion of radiative and nonradiative recombination channels, details on analytic and numerical simulations, supplemental experimental data on the all-dielectric spatial control in NWs (PDF)

\section{AUTHOR INFORMATION}

\section{Corresponding Author}

*E-mail: dominique.bougeard@ur.de.

\section{ORCID $\odot$}

Florian Dirnberger: 0000-0002-2932-2850

Christoph Lange: 0000-0002-2134-6612

Tobias Korn: 0000-0003-4808-391X

José Sánchez-Gil: 0000-0002-5370-3717

Dominique Bougeard: 0000-0003-3793-2787

Notes

The authors declare no competing financial interest.

\section{ACKNOWLEDGMENTS}

We gratefully acknowledge financial support by the German Research Foundation (DFG) via SFB 689 and 1277 Project A01. J.A.S.-G. and D.R.A. acknowledge partial financial support from the Spanish Ministerio de Ciencia, Innovación y Universidades through Grants NANOTOPO (FIS2017-
91413-EXP) and MELODIA (PGC2018-095777-B-C21, MCIU/AEI/FEDER, UE), and through the FPU Ph.D. Fellowship FPU15/03566.

\section{REFERENCES}

(1) Qian, F.; Gradečak, S.; Li, Y.; Wen, C.-Y.; Lieber, C. M. Nano Lett. 2005, 5, 2287-2291.

(2) Krogstrup, P.; Jørgensen, H. I.; Heiss, M.; Demichel, O.; Holm, J. V.; Aagesen, M.; Nygard, J.; Fontcuberta i Morral, A. Nat. Photonics 2013, 7, 306.

(3) Saxena, D.; Mokkapati, S.; Parkinson, P.; Jiang, N.; Gao, Q.; Tan, H. H.; Jagadish, C. Nat. Photonics 2013, 7, 963.

(4) Peng, K.; Parkinson, P.; Fu, L.; Gao, Q.; Jiang, N.; Guo, Y.-N.; Wang, F.; Joyce, H. J.; Boland, J. L.; Tan, H. H.; Jagadish, C.; Johnston, M. B. Nano Lett. 2015, 15, 206-210.

(5) Su, Y.; Liu, C.; Brittman, S.; Tang, J.; Fu, A.; Kornienko, N.; Kong, Q.; Yang, P. Nat. Nanotechnol. 2016, 11, 609.

(6) Parkinson, P.; Joyce, H. J.; Gao, Q.; Tan, H. H.; Zhang, X.; Zou, J.; Jagadish, C.; Herz, L. M.; Johnston, M. B. Nano Lett. 2009, 9, 3349-3353.

(7) Wang, F.; Gao, Q.; Peng, K.; Li, Z.; Li, Z.; Guo, Y.; Fu, L.; Smith, L. M.; Tan, H. H.; Jagadish, C. Nano Lett. 2015, 15, 3017-3023.

(8) Demichel, O.; Heiss, M.; Bleuse, J.; Mariette, H.; Fontcuberta i Morral, A. Appl. Phys. Lett. 2010, 97, 201907.

(9) Nguyen, H. P. T.; Djavid, M.; Woo, S. Y.; Liu, X.; Connie, A. T.; Sadaf, S.; Wang, Q.; Botton, G. A.; Shih, I.; Mi, Z. Sci. Rep. 2015, 5, 7744.

(10) Dan, Y.; Seo, K.; Takei, K.; Meza, J. H.; Javey, A.; Crozier, K. B. Nano Lett. 2011, 11, 2527-2532.

(11) Speckbacher, M.; Treu, J.; Whittles, T. J.; Linhart, W. M.; Xu, X.; Saller, K.; Dhanak, V. R.; Abstreiter, G.; Finley, J. J.; Veal, T. D.; Koblmüller, G. Nano Lett. 2016, 16, 5135-5142.

(12) Yong, C. K.; Noori, K.; Gao, Q.; Joyce, H. J.; Tan, H. H.; Jagadish, C.; Giustino, F.; Johnston, M. B.; Herz, L. M. Nano Lett. 2012, 12, 6293-6301.

(13) Lodahl, P.; Mahmoodian, S.; Stobbe, S. Rev. Mod. Phys. 2015, 87, 347-400.

(14) Mayer, B.; Rudolph, D.; Schnell, J.; Morkötter, S.; Winnerl, J.; Treu, J.; Müller, K.; Bracher, G.; Abstreiter, G.; Koblmüller, G.; Finley, J. Nat. Commun. 2013, 4, 2931.

(15) Oulton, R. F.; Sorger, V. J.; Zentgraf, T.; Ma, R.-M.; Gladden, C.; Dai, L.; Bartal, G.; Zhang, X. Nature 2009, 461, 629.

(16) Furthmeier, S.; Dirnberger, F.; Hubmann, J.; Bauer, B.; Korn, T.; Schüller, C.; Zweck, J.; Reiger, E.; Bougeard, D. Appl. Phys. Lett. 2014, 105, 222109.

(17) Dirnberger, F.; Kammermeier, M.; König, J.; Forsch, M.; Faria Junior, P. E.; Campos, T.; Fabian, J.; Schliemann, J.; Schüller, C.; Korn, T.; Wenk, P.; Bougeard, D. Appl. Phys. Lett. 2019, 114, 202101.

(18) Furthmeier, S.; Dirnberger, F.; Gmitra, M.; Bayer, A.; Forsch, M.; Hubmann, J.; Schüller, C.; Reiger, E.; Fabian, J.; Korn, T.; Bougeard, D. Nat. Commun. 2016, 7, 12413.

(19) Anttu, N. Nanotechnology 2019, 30, 104004.

(20) Abujetas, D. R.; Paniagua-Domínguez, R.; Sánchez-Gil, J. A. ACS Photonics 2015, 2, 921-929.

(21) Hildebrand, O.; Goebel, E. O.; Romanek, K. M.; Weber, H.; Mahler, G. Phys. Rev. B: Condens. Matter Mater. Phys. 1978, 17, 47754787.

(22) Hayamizu, Y.; Yoshita, M.; Takahashi, Y.; Akiyama, H.; Ning, C. Z.; Pfeiffer, L. N.; West, K. W. Phys. Rev. Lett. 2007, 99, 167403.

(23) Ahtapodov, L.; Todorovic, J.; Olk, P.; Mjåland, T.; Slåttnes, P.; Dheeraj, D. L.; van Helvoort, A. T. J.; Fimland, B.-O.; Weman, H. Nano Lett. 2012, 12, 6090-6095.

(24) Boland, J. L.; Casadei, A.; Tütüncüoglu, G.; Matteini, F.; Davies, C. L.; Jabeen, F.; Joyce, H. J.; Herz, L. M.; Fontcuberta Morral, A.; Johnston, M. B. ACS Nano 2016, 10, 4219-4227.

(25) Fickenscher, M. A.; Jackson, H. E.; Smith, L. M.; Yarrison-Rice, J. M.; Kang, J. H.; Paiman, S.; Gao, Q.; Tan, H. H.; Jagadish, C. Appl. Phys. Lett. 2011, 99, 263110. 
(26) Feldmann, J.; Peter, G.; Göbel, E. O.; Dawson, P.; Moore, K.; Foxon, C.; Elliott, R. J. Phys. Rev. Lett. 1987, 59, 2337-2340.

(27) Pelton, M. Nat. Photonics 2015, 9, 427.

(28) Noda, S.; Fujita, M.; Asano, T. Nat. Photonics 2007, 1, 449.

(29) Englund, D.; Fattal, D.; Waks, E.; Solomon, G.; Zhang, B.; Nakaoka, T.; Arakawa, Y.; Yamamoto, Y.; Vučković, J. Phys. Rev. Lett. 2005, 95, 013904.

(30) Akselrod, G. M.; Argyropoulos, C.; Hoang, T. B.; Ciracì, C.; Fang, C.; Huang, J.; Smith, D. R.; Mikkelsen, M. H. Nat. Photonics 2014, 8, 835 .

(31) Bleuse, J.; Claudon, J.; Creasey, M.; Malik, N. S.; Gérard, J.-M.; Maksymov, I.; Hugonin, J.-P.; Lalanne, P. Phys. Rev. Lett. 2011, 106, 103601.

(32) Grzela, G.; Paniagua-Domínguez, R.; Barten, T.; Fontana, Y.; Sánchez-Gil, J. A.; Gómez Rivas, J. Nano Lett. 2012, 12, 5481-5486.

(33) Tong, L.; Lou, J.; Mazur, E. Opt. Express 2004, 12, 1025-1035.

(34) Paniagua-Dominguez, R.; Grzela, G.; Rivas, J. G.; Sánchez-Gil, J. A. Nanoscale 2013, 5, 10582-10590.

(35) Bulgarini, G.; Reimer, M. E.; Zehender, T.; Hocevar, M.; Bakkers, E. P. A. M.; Kouwenhoven, L. P.; Zwiller, V. Appl. Phys. Lett. 2012, 100, 121106.

(36) Birowosuto, M. D.; Zhang, G.; Yokoo, A.; Takiguchi, M.; Notomi, M. Opt. Express 2014, 22, 11713-11726.

(37) Brenny, B. J.; Abujetas, D. R.; van Dam, D.; Sánchez-Gil, J. A.; Rivas, J. G.; Polman, A. ACS Photonics 2016, 3, 677-684.

(38) Kuznetsov, A. I.; Miroshnichenko, A. E.; Brongersma, M. L.; Kivshar, Y. S.; Luk'yanchuk, B. Science 2016, 354, aag2472. 